UFIFT-HEP-98-14

\title{
Family Symmetry and Neutrino Mixing
}

\author{
John K. Elwood ${ }^{2}$, Nikolaos Irges $^{1}$ and Pierre Ramond ${ }^{1}$ 用 \\ ${ }^{1}$ Institute for Fundamental Theory, \\ Department of Physics, University of Florida \\ Gainesville FL 32611, USA \\ ${ }^{2}$ Department of Physics, Kent State University, \\ Kent, Ohio 44242, USA
}

\begin{abstract}
The observed quark hierarchies suggest a simple family symmetry. Generalized to leptons through grand-unified quantum numbers, it produces a neutrino mixing matrix with order-one $\nu_{\mu}-\nu_{\tau}$ mixing, and order- $\lambda^{3} \nu_{e}-\nu_{\mu}$ and $\nu_{e}-\nu_{\tau}$ mixings. The intrafamily hierarchy and observed neutrino mass differences together require this symmetry to be anomalous, suggesting through the Green-Schwarz mechanism a string or M-theory origin for the symmetry.
\end{abstract}

June 1998

*e-mail address jelwood@phys.ufl.edu

†e-mail address irges@phys.ufl.edu

${ }^{\ddagger}$ e-mail address ramond@phys.ufl.edu 


\section{INTRODUCTION}

The Yukawa sector of the Standard Model is clearly its most poorly understood aspect, with a number of undetermined masses and mixings. Its extension to include low energy supersymmetry, however, brings about several conceptual simplifications including the unification of its gauge couplings and the equality of the bottom quark and tau lepton Yukawa couplings at a scale $M_{G U T} \sim 10^{16} \mathrm{GeV}$. Further regularities in the Yukawa sector are best appreciated by using the Wolfenstein parametrization of the CKM matrix [1],

$$
\left(\begin{array}{ccc}
1 & \lambda & \lambda^{3} \\
\lambda & 1 & \lambda^{2} \\
\lambda^{3} & \lambda^{2} & 1
\end{array}\right),
$$

in terms of the Cabibbo angle $\lambda$, with all prefactors of order one. Extended to the quark and charged lepton mass ratios, it reveals both an interfamily hierarchy

$$
\frac{m_{u}}{m_{t}} \sim \lambda^{8} \frac{m_{c}}{m_{t}} \sim \lambda^{4} \quad ; \quad \frac{m_{d}}{m_{b}} \sim \lambda^{4} \quad \frac{m_{s}}{m_{b}} \sim \lambda^{2} ; \frac{m_{e}}{m_{\tau}} \sim \lambda^{4} \quad \frac{m_{\mu}}{m_{\tau}} \sim \lambda^{2}
$$

and an intrafamily hierarchy

$$
\frac{m_{b}}{m_{t}} \sim \lambda^{3} \frac{m_{b}}{m_{\tau}} \sim 1
$$

valid at the scale $M \sim M_{G U T}$. Such relations become apparent if low energy supersymmetry is realized.

Below we argue for a simple explanation: the interfamily hierarchy points to the existence of at least one $U(1)$ family symmetry beyond the Standard Model. In addition, the intrafamily hierarchy, together with the recent neutrino data [4], requires one of these to be anomalous [2,3].

In our approach, the Standard Model is an effective field theory with cut-off $M \sim M_{G U T}$. Standard Model invariants may not appear in the superpotential unless they also have zero charge with respect to any additional symmetries; in this way, invariants with non-zero charges will be present only as higher dimension operators, leading to the suppression of their couplings. Thus, the exponents that appear in the hierarchies can be directly related to the

charges of the accompanying SM invariants [5.6]. This letter addresses only the origin of the Cabibbo suppressions of the Yukawa couplings, not the supersymmetry nor the electroweak breaking mechanisms. 


\section{INTERFAMILY HIERARCHY}

To study the interfamily hierarchy, it suffices to consider the relative strengths of the Yukawa couplings within each charge sector separately. In the down quark sector, the observed hierarchy allows us to reconstruct part of the $3 \times 3$ Yukawa matrix that labels the strength of the operators $\mathbf{Q}_{i} \overline{\mathbf{d}}_{j} H_{d}$. Assuming the matrices that diagonalize the Yukawa couplings are, like the CKM matrix, nearly diagonal, the mass ratios determine its (11) and (22) elements to be of order $\lambda^{4}$ and $\lambda^{2}$, respectively, as compared to the (33) element, which we take to be of order of one. 3 Our first assumption is that both matrices that make up the CKM matrix have the same Cabibbo structure (no alignment网). Then, the upper off-diagonal elements are determined by the CKM matrix, yielding the exponents

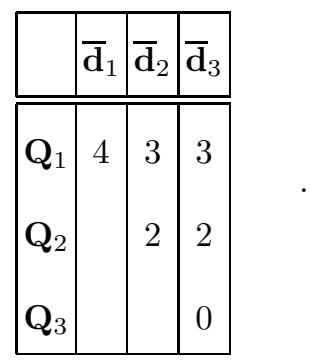

The orders of magnitude of elements below the diagonal are not determined by phenomenology. Our second assumption is that the exponents are determined by the charges of the operators under a family symmetry $Y_{F}$. The exponents in the $(i j)$ entry, for example, would be proportional to $\left(x_{\mathbf{Q}_{i}}+x_{\overline{\mathbf{d}}_{j}}+x_{H_{d}}\right)$. The charge of $H_{d}$ does not affect the interfamily patterns. This origin for the exponents implies sum rules between them $\left(n_{i j}+n_{j i}=n_{i i}+n_{j j}\right)$ [3], leading to the full matrix:

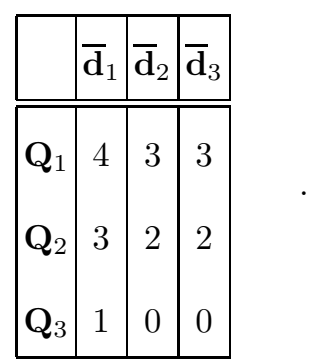

\footnotetext{
${ }^{\S}$ Since here we are interested only in the interfamily hierarchy, we neglect for the time being an overall factor that may multiply these elements.

${ }^{* *}$ This means our model must rely on a supersymmetry-breaking mechanism that generates no large FCNC effects, whose discussion is beyond the scope of this letter.
} 
When an operator's charge prevents it from appearing in the superpotential as a holomorphic invariant [7], we say that the operator develops a "supersymmetric zero". Unless otherwise specified, we will assume throughout this paper, as we just did, that there are no supersymmetric zeros. From the above matrix, we can read off the charges of the $\overline{\mathbf{d}}_{i}$ and $\mathbf{Q}_{i}$ :

$$
\begin{gathered}
\left(\overline{\mathbf{d}}_{1}, \overline{\mathbf{d}}_{2}, \overline{\mathbf{d}}_{3}\right)=-\left(\frac{1}{3}\right)(2,-1,-1) \\
\left(\mathbf{Q}_{1}, \mathbf{Q}_{2}, \mathbf{Q}_{3}\right)=-\left(\frac{1}{3}\right)(4,1,-5) .
\end{gathered}
$$

Since we are discussing only the interfamily hierarchies, we need consider only the family-traceless charges. Suggestively, we can express these in terms of baryon number as

$$
\begin{gathered}
\left(\overline{\mathbf{d}}_{1}, \overline{\mathbf{d}}_{2}, \overline{\mathbf{d}}_{3}\right)=B(2,-1,-1), \\
\left(\mathbf{Q}_{1}, \mathbf{Q}_{2}, \mathbf{Q}_{3}\right)=B(2,-1,-1)-2(1,0,-1) .
\end{gathered}
$$

In the charge $2 / 3$ sector, the (11) and (22) elements have exponents 8 and 4 respectively, and using the CKM matrix, we obtain:

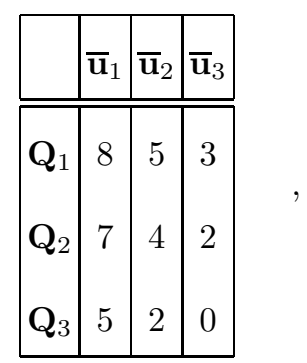

where the entries below the diagonal are determined by sum rules. We arrive of course at exactly the same charges for the $\mathbf{Q}_{i}$, but find also the $\overline{\mathbf{u}}_{i}$ charge assignment

$$
\left(\overline{\mathbf{u}}_{1}, \overline{\mathbf{u}}_{2}, \overline{\mathbf{u}}_{3}\right)=B(2,-1,-1)-2(1,0,-1)
$$

Thus, the family dependent symmetry acting upon the quark sector may be written

$$
Y_{F}=B(2,-1,-1)-2 \eta(1,0,-1)
$$

where $\eta=1$ for both $\mathbf{Q}$ and $\overline{\mathbf{u}}$ and $\eta=0$ for $\overline{\mathbf{d}}$. That both $\mathbf{Q}$ and $\overline{\mathbf{u}}$ possess the same $\eta$ charge is reminiscent of the $S U(5)$ charge patterns, where the chiral fermions are split into $\overline{\mathbf{5}}=(L, \overline{\mathbf{d}})$ and $\mathbf{1 0}=(\mathbf{Q}, \overline{\mathbf{u}}, \bar{e})$. Our third assumption 
uses these Grand Unified patterns to assign family charges to the leptons: we flesh out the multiplets by assigning the $\bar{e}$ singlet a value $\eta=1$ and the $L$ doublet $\eta=0$, and generalize the factor $B$ appearing in Eq. (II.9) to its $S O(10)$ analog $(B-L)$. Note that $\eta$, on the other hand, is outside of $S O(10)$. The quark and lepton charges may then be succinctly written:

$$
Y_{F}=(B-L)(2,-1,-, 1)-2 \eta(1,0,-1)
$$

The interfamily exponents of the Yukawa matrix associated with the operator $L_{i} \bar{e}_{j} H_{d}$ follow:

\begin{tabular}{|l|l|l|l|}
\hline & $\overline{\mathrm{e}}_{1}$ & $\bar{e}_{2}$ & $\bar{e}_{3}$ \\
\hline \hline$L_{1}$ & 4 & 5 & 3 \\
$L_{2}$ & 1 & 2 & 0 \\
$L_{3}$ & 1 & 2 & 0 \\
\hline
\end{tabular}

Its diagonalization yields the lepton interfamily hierarchy

$$
\frac{m_{e}}{m_{\tau}} \sim \lambda^{4}, \quad \frac{m_{\mu}}{m_{\tau}} \sim \lambda^{2}
$$

not inconsistent with phenomenology, as well as the contribution to the lepton mixing matrix from the rotation of the left-handed lepton doublet:

$$
\left(\begin{array}{ccc}
1 & \lambda^{3} & \lambda^{3} \\
\lambda^{3} & 1 & 1 \\
\lambda^{3} & 1 & 1
\end{array}\right)
$$

As $Y_{F}$ contains $B-L$, it is natural to introduce three families of right handed neutrinos $\bar{N}_{i}$. Before assigning them $Y_{F}$ charges, we note that certain predictions associated with neutrino phenomenology are completely independent of the charges of the $\bar{N}_{s}$. The neutrino mixing matrix, for example, is uniquely determined by the charges of the MSSM fields [8]. This is a result of its seesaw [9] origin, as can be seen via the following simple argument. Since the right-handed neutrino Majorana mass matrix is symmetric, it may be written $Y_{i j}^{(0)}=\bar{N}_{i} \bar{N}_{j}$, where $\bar{N}_{i}$ and $\bar{N}_{j}$ are vectors depending only on the antineutrino charges. The matrix coupling right-handed neutrinos to the standard model, on the other hand, is written $Y_{i j}^{(\nu)}=H_{u} L_{i} \bar{N}_{j}$, where $H_{u} L_{i}$ is a vector independent of the right-handed charges. Taking $U^{0}$ to be the matrix that diagonalizes $Y^{[0]}$, 


$$
Y^{(0)}=U^{0} D^{0}\left(U^{0}\right)^{T}
$$

with $D^{0}$ a diagonal matrix, the effective neutrino mixing matrix after the seesaw is given by:

$$
\hat{Y}^{(\nu)}=-\left(Y^{(\nu)} U^{0}\right)\left(D^{0}\right)^{-1}\left(Y^{(\nu)} U^{0}\right)^{T}
$$

Because of the form of Eq. (II.14), a cancellation of $\bar{N}_{i}$ charges results, and one discovers that

$$
\hat{Y}_{i j}^{(\nu)}=-H_{u}^{2} L_{i} L_{j} \quad .
$$

The MNS neutrino mixing matrix [10] therefore depends only on the mixing of the $L_{i}$, a fact already noted in Refs. [11,12]. Thus, both the neutrino mass matrix and the MNS mixing matrix appearing in the leptonic charged current are determined by the $L_{i}$ charges, and the MNS mixing matrix will be of the form given in Eq. (II.12).

$$
\mathcal{U}_{M N S} \sim\left(\begin{array}{ccc}
1 & \lambda^{3} & \lambda^{3} \\
\lambda^{3} & 1 & 1 \\
\lambda^{3} & 1 & 1
\end{array}\right)
$$

This implies a small (order $\lambda^{3}$ ) mixing of the electron neutrino with the $\mu$ and $\tau$ species, and mixing between the $\mu$ and $\tau$ neutrinos of order one [13]. Remarkably enough, this mixing pattern is precisely the one suggested by the non-adiabatic MSW [14] explanation of the solar neutrino deficit and by the oscillation interpretation of the reported anomaly in atmospheric neutrino fluxes [4, 15, 16]. It is important to stress that this mixing matrix is a generic prediction of such models, and depends only on standard model charges already fixed by phenomenology. The neutrino masses, on the other hand, depend on the origin of the intrafamily hierarchy.

\section{INTRAFAMILY HIERARCHY}

The intrafamily hierarchy in the quark sector suggests that a family-independent symmetry is not the end of the story. Recall that the ratio of third family quarks, $m_{b} / m_{t}$, is of order $\lambda^{3}$. Since both $\cot \beta$ and the Yukawa entries conspire to produce this suppression, there are two extreme possibilities.

- The first possibility is that $Y_{b}$ and $Y_{t}$ are of the same order, with $\cot \beta$ responsible for the suppression. With a tree-level top quark mass, achieving $Y_{b}$ and $Y_{t}$ of the same order requires that the $Y_{F}$ charge of the $\mu$-term, $H_{u} H_{d}$, be $Y_{F}^{[\mu]}=-6$. But avoiding anomalies such as $\operatorname{Tr}\left[Y Y Y_{F}\right]$ and $\operatorname{Tr}\left[S U(2) S U(2) Y_{F}\right]$ forces the $Y_{F}$ charge to be vector-like

on the Higgs doublets, so that $Y_{F}^{[\mu]}=0$. Hence $Y_{b} \sim Y_{t}$ requires $Y_{F}$ to be anomalous (The Green-Schwarz mechanism 
cannot be invoked since $\left.\operatorname{Tr}\left[S U(3) S U(3) Y_{F}\right]=0\right)$. Furthermore, we shall soon see that a family-traceless $Y_{F}$ cannot reproduce neutrino phenomenology.

To proceed, we need to assign $Y_{F}$ charges to the right-handed neutrinos $\bar{N}$. Since $\eta$ is contained in $E_{6}$, we give the $\bar{N}$ fields their $E_{6}$ value, $\eta=2$, which yields $Y_{F}\left(\bar{N}_{i}\right)=(-2,-1,3)$. One obtains an $\bar{N}_{i} \bar{N}_{j}$ Majorana mass matrix with family dependence

\begin{tabular}{|c|c|c|c|}
\hline & $\bar{N}_{1}$ & $\bar{N}_{2}$ & $\bar{N}_{3}$ \\
\hline \hline $\bar{N}_{1}$ & 4 & 3 & $S Z$ \\
$\bar{N}_{2}$ & 3 & 2 & $S Z$ \\
$\bar{N}_{3}$ & $S Z$ & $S Z$ & $S Z$ \\
\hline
\end{tabular}

where 'the SZ' stand for 'supersymmetric zeros' due to negative charges. With a null row, this matrix has a zero eigenvalue, and the third family neutrino drops out of the seesaw mechanism. We are then left with two light species of neutrinos, with masses $v_{u}^{2} \lambda^{6} / M$ and $v_{u}^{2} \lambda^{12} / M$. This situation is inconsistent with the combined set of atmospheric and solar neutrino data. The predictions can be made to fit any one experiment, however, but only if $M$ is of order $10^{12} \mathrm{GeV}$, suppressed by four orders of magnitude with respect to $M_{G U T}$. There is no mechanism in our model to effect such a suppression. We conclude that the family-traceless, non-anomalous $Y_{F}$ symmetry must be extended by adding a family-independent piece, hereafter called $X$.

- We turn now to the alternate possibility, $Y_{b} \sim \lambda^{3} Y_{t}$ and $\cot \beta$ of order 1 , where the suppression $\lambda^{3}$ comes from the family-independent piece $X$. The total flavor symmetry is now

$$
Y_{X} \equiv X+Y_{F}
$$

To consider the implications of anomalies involving our family-independent symmetry, we define the mixed anomaly coefficients of $Y_{X}$ with the Standard Model gauge fields by

$$
C_{G_{i}}=\operatorname{Tr}\left[G_{i} G_{i} Y_{X}\right]
$$

The $C_{G_{i}}$ satisfy the following relations:

$$
\begin{gathered}
C_{Y}+C_{\text {weak }}-\frac{8}{3} C_{\text {color }}=6\left(X^{[e]}-X^{[d]}\right)+2 X^{[\mu]}, \\
C_{\text {color }}=3\left(X^{[u]}+X^{[d]}\right)-3 X^{[\mu]},
\end{gathered}
$$


where $X^{[u, e, d, \mu]}$ are the $X$ charges of the operators $\mathbf{Q}_{i} \overline{\mathbf{u}}_{j} H_{u}, L_{i} \bar{e}_{j} H_{d}, \mathbf{Q}_{i} \overline{\mathbf{d}}_{j} H_{d}$, and $H_{u} H_{d}$, respectively. It is precisely these charges $X^{[e]}, X^{[d]}$, and $X^{[\mu]}$ that determine the intrafamily hierarchies $m_{b} / m_{t}$ and $m_{\tau} / m_{b}$. Let us set

$$
\frac{m_{b}}{m_{t}} \sim \cot \beta \lambda^{P_{b t}}, \frac{m_{\tau}}{m_{b}} \sim \lambda^{P_{\tau b}} .
$$

Then one finds that Eqs. (III.19) and (III.20) above may be rewritten as

$$
C_{Y}+C_{\text {weak }}-2 C_{\text {color }}=-2\left(P_{b t}+3 P_{\tau b}+6\right),
$$

where we have used the fact that the top quark Yukawa coupling appears at tree level, and therefore that $Y^{[u]}=0$. The data suggest $P_{b t}=3$ and $P_{\tau b}=0$, which through Eq. (III.22) tells us that our new symmetry $Y_{X}$ must be anomalous. The only consistent way to build a model with such an anomalous $U(1)$ is the use of the four dimensional version of the Green-Schwarz anomaly cancellation mechanism [17]. We take the family-independent $X$ acting on the chiral fields to be a linear combination of a universal piece and of the two $E_{6}$ charges, $V, V^{\prime}$, defined through

$$
E_{6} \supset S O(10) \times U(1)_{V^{\prime}} \quad ; \quad S O(10) \supset S U(5) \times U(1)_{V} .
$$

Across the Higgs doublets, the $X$ symmetry is taken to be vector-like, a necessary condition if the three $U(1)$ symmetries comprising $Y_{X}$ are gauged separately [12]. These choices yield $X^{[d]}=X^{[e]}$, and $X^{[u]}=X\left(L H_{u} \bar{N}\right)=0$. The Green-Schwarz structure has the added benefit of producing the correct value of the Weinberg angle at cut-off 18,2, 2, 3]:

$$
\cot ^{2} \theta_{w}=\frac{C_{Y}}{C_{\text {weak }}}=\frac{5}{3}
$$

There still remains the non-zero anomaly $\left(Y Y_{X} Y_{X}\right)$, which can be canceled by three families of standard model vector-like representations $\mathbf{5}+\overline{\mathbf{5}}$ of $S U(5)$. With this addition made, the remaining anomaly structure is consistent with the Green-Schwarz cancellation mechanism. We get

$$
\frac{m_{b}}{m_{t}} \sim \cot \beta \lambda^{-\left(C_{\text {color }}+18\right) / 3}, \frac{m_{\tau}}{m_{b}} \sim 1
$$

and agreement with the data is achieved for $C_{\text {color }}=-27$.

We can now specify the form of the matrices involving right-handed neutrinos:

$$
Y^{(0)} \sim M \lambda^{-2 X^{[\bar{N}]}-2}\left(\begin{array}{ccc}
\lambda^{6} & \lambda^{5} & \lambda \\
\lambda^{5} & \lambda^{4} & 1 \\
\lambda & 1 & \lambda^{-4}
\end{array}\right) \quad ; \quad Y^{(\nu)} \sim v_{u}\left(\begin{array}{ccc}
\lambda^{8} & \lambda^{7} & \lambda^{3} \\
\lambda^{5} & \lambda^{4} & 1 \\
\lambda^{5} & \lambda^{4} & 1
\end{array}\right)
$$


where $Y^{(0)}$ is the $\overline{N N}$ Majorana mass matrix and $Y^{[\nu]}$ the matrix coupling $L_{i}$ to $\bar{N}_{j}$. Note that, to appear in the superpotential only as holomorphic quadratic mass terms, the $X$-charge of the $\bar{N}$ s must be negative half odd integers.

After the seesaw, we have the actual neutrino mass matrix

$$
\hat{Y}^{(\nu)} \sim \frac{v_{u}^{2} \lambda^{2 X^{[\bar{N}}}+6}{M}\left(\begin{array}{ccc}
\lambda^{6} & \lambda^{3} & \lambda^{3} \\
\lambda^{3} & 1 & 1 \\
\lambda^{3} & 1 & 1
\end{array}\right),
$$

which produces light neutrinos with masses

$$
m_{\nu_{e}} \sim \frac{v_{u}^{2} \lambda^{2 X^{[\bar{N}]}+12}}{M} ; m_{\nu_{\mu}} \sim m_{\nu_{\tau}} \sim \frac{v_{u}^{2} \lambda^{2 X^{[\bar{N}]}+6}}{M} .
$$

The mass splitting between $\nu_{e}$ and the other two neutrinos is $\Delta m_{\nu_{\mu}-\nu_{e}}^{2} \sim 10^{-5} \mathrm{eV}^{2}$, consistent with the non-adiabatic MSW solution to the solar neutrino problem if $X^{[\bar{N}]}=-9 / 2$ and $M \sim M_{G U T}$. To check agreement with the atmospheric neutrino data, we must know the mass splitting between $\nu_{\mu}$ and $\nu_{\tau}$, but this can be predicted only with a theory for the prefactors. Interestingly, as shown in Ref. [12], prefactors of order 1 produce $\Delta m_{\nu_{\tau}-\nu_{\mu}}^{2} \sim 0.07 \mathrm{eV}^{2}$, so that the atmospheric data may be explained by the same solution that accommodates the solar neutrino data without any fine tuning. Moreover, this solution requires $M$ to be of order $M_{G U T}$ as well, and drives the mixing angle to maximal, in agreement with recent experimental results [4, 15, 16].

As mentioned before, it is possible to gauge separately the three symmetries that make up $Y_{X}$. The analysis proceeds much as that above, but in this case $X^{[d]}=-3$ instead of $X^{[d]}=-9$. Also, as shown in [12], the extra anomaly conditions fix all the charges of the $\mu$ term to zero, and the analysis of the vacuum 19 in which all three symmetries are broken at the same scale favors $X^{[\bar{N}]}=-3 / 2$. Remarkably, it is precisely this charge assignment, corresponding to $X^{[\bar{N}]}=-9 / 2$ when the three symmetries are combined into a single gauged symmetry $Y_{X}$, that leads to a fit of the neutrino data with $M \sim M_{G U T}$. We refer the interested reader to Ref. [12] for more details.

\section{ACKNOWLEDGEMENTS}

JE would like to thank the Institute for Fundamental Theory at the University of Florida for its hospitality during the completion of this work, and Kent State University for a summer grant in support of the work. NI and PR are supported in part by the United States Department of Energy under grant DE-FG02-97ER41029. 
[1] L. Wolfenstein, Phys. Rev. Lett. 51, 1945 (1983).

[2] L. Ibáñez and G. G. Ross, Phys. Lett. B332, 100 (1994).

[3] P. Binétruy and P. Ramond, Phys. Lett. B350, 49 (1995).

[4] The Super-Kamiokande Collaboration, hep-ex/9805006 v2.

[5] C. Froggatt and H. B. Nielsen Nucl. Phys. B147, 277 (1979).

[6] P. Ramond, R.G. Roberts and G.G. Ross, Nucl. Phys. B406 (1993)

[7] M. Leurer, Y. Nir, N. Seiberg, Nucl. Phys. B398, 319 (1993), Phys. Lett. B309, 337 (1993).

[8] A. Rasin and J.P. Silva, Phys. Rev. D49, 20 (1994); Y. Grossman and Y. Nir, Nucl. Phys. B448, 30 (1995).

[9] M. Gell-Mann, P. Ramond, and R. Slansky in Sanibel Talk, CALT-68-709, Feb 1979, and in Supergravity (North Holland, Amsterdam 1979). T. Yanagida, in Proceedings of the Workshop on Unified Theory and Baryon Number of the Universe, KEK, Japan, 1979.

[10] Z. Maki, M. Nakagawa,and S. Sakata, Prog. Theo. Phys. 28, 247 (1962).

[11] J. K. Elwood, N. Irges and P. Ramond, Phys. Lett. B413, 322 (1997).

[12] N. Irges, S. Lavignac and P. Ramond, hep-ph/9802334, to be published in Phys. Rev. D.

[13] J. A. Harvey, P. Ramond and D. B. Reiss, Nucl. Phys. B199, 223 (1982); C. Carone and M. Sher, Phys. Lett. B420, 83(1998); Carl H. Albright, K.S. Babu, and S.M. Barr, contribution submitted to NEUTRINO 98 conference, hep-ph/9805226; M. Bando, T. Kugo, and K. Yoshioka, Phys. Rev. Lett. 80, 3004 (1998).

[14] L. Wolfenstein, Phys. Rev. D17, 2369 (1978); S. Mikheyev and A. Yu Smirnov, Nuovo Cim. 9C, 17 (1986).

[15] E. Kearns, talk at the ITP conference on Solar Neutrinos: News about SNUs, December 2-6 1997.

[16] S.M. Kasahara et al., Phys. Rev. D55, 5282 (1997).

[17] M. Green and J. Schwarz, Phys. Lett. B149, 117 (1984).

[18] L. Ibáñez, Phys. Lett. B303, 55 (1994).

[19] N. Irges and S. Lavignac, Phys. Lett. B424, 293 (1998). G. Cleaver, M. Cvetic, J. R. Espinosa, L. Everett, P. Langacker, CERN-TH-97-338, hep-th/9711178. 